\title{
Analysis of ECG Signals Recorded Using Different Stimuli from Patients in Intensive Care Unit: A Case Study
}

\author{
${ }^{1}$ Çiğdem Gülüzar Altıntop, ${ }^{1}$ Fatma Latifoğlu*, ${ }^{3}$ Mehmet Akif Yazar, ${ }^{2}$ Aynur Karayol Akın, ${ }^{1}$ Ramis İleri \\ ${ }^{1}$ Faculty of Engineering, Department of Biomedical Engineering Erciyes University, Turkey \\ ${ }^{2}$ Faculty of Medicine, Department of Anesthesiology and Reanimation, Erciyes University, Turkey \\ ${ }^{3}$ Department of Anesthesiology and Reanimation, Konya Training and Research Hospital, Turkey
}

\begin{abstract}
Intensive Care Units (ICUs) are more difficult and complex areas of hospital as treatment and care. In these units, the patients are monitored continuously with a bedside monitor for respiration, $\mathrm{O}_{2}$ saturation and pulse information. However, this information, which is periodically noted on the patient observation papers, may not represent a definite diagnose/follow up about the patient's health condition in all times. Therefore, in order to assist the physician in these units where the diagnosis/follow up is important, attributes are extracted from ECG signals easy-to-obtain by using signal processing methods. ECG signals were obtained from 3 patients at different days. Attributes were analyzed statistically to see if the patient reacted to oral/touch stimuli and to monitor his / her health condition. As a result, it was possible to evaluate the coma patients' response to stimuli and to follow-up for improving physiological well-being using ECG signals.
\end{abstract}

Key words: intensive care units, electrocardiogram, heart rate variability, sound stimuli, patient follow-up

\section{Introduction}

Consciousness is that the person is aware of himself/herself and what is happening around him and can adapt to new stimuli. Evaluation of the patient's consciousness state is important for neurological evaluation. Consciousness is a factor that affects the stages of patient evaluation and treatment. Neurological assessment requires patient assistance because motor and sensory evaluation cannot be performed for patients who are unable to respond to the given commands [1-3].

Consciousness level is not directly measured. It is measured according to the patient's response to stimuli. In the assessment of consciousness, firstly oral stimuli are given to the patient (such as how he/she is or asking for personal information). If the patient is unable to speak, he/she may be asked to respond with head shaking and eye movements. The patient's motor response is observed with intense but non-destructive painful stimuli such as trapezeus compression $[1,3]$. Consciousness levels are shown in Figure 1. The changes between these levels may occur suddenly or slowly. In coma, the patient's eyes are closed, do not response to environmental stimuli and do not have spontaneous movements [3].

Electrocardiography is the process of recording the electrical activity of the heart for a period of time using electrodes placed on the body. These electrodes detect small electrical changes in the

*Corresponding author: Address: Faculty of Engineering, Department of Biomedical Engineering Erciyes University, TURKEY, E-mail address: flatifoglu@ erciyes.edu.tr, Phone: +9003522076666 
skin caused by the depolarizing heart muscle at each heartbeat. In the conventional 12-lead Electrocardiogram (ECG), ten electrodes are placed on the patient's chest surface. Then the magnitude of the electrical potential of the heart is measured at twelve different derivations and recorded over a period (usually 10 seconds). In this way, the overall magnitude and direction of cardiac electrical depolarization is captured. As a result, the ECG signal is generated as a graph of time versus voltage.The ECG can be used to measure heart rate and rhythm, the size and location of the heart chambers, any damage to the heart muscle cells or conduction system, the effects of heart medications [4]

Clinical scales are susceptible to problems of sensitivity, specificity, subjectivity and interrater reliability. This leads to a misdiagnosis of up to $40 \%$ and consequences associated with inappropriate treatment decisions [5]. Wieser et al. reported that they used objective measurements including physiological and neurological signals to measure the consciousness status of 8 patients ( 5 females, 3 males). They conducted a linear retrospective regression analysis and concluded that the 13 variables obtained were enough to define $74.7 \%$ of the variability in the score. They used ECG signals in their studies. The frequency bands of the ECG were analyzed, and RMS and standard deviation values were calculated [5].

In the literature, ECG signals have been obtained to determine the stress experienced by drivers [6] or to examine stress levels [7]. Because ECG signals are easier to obtain and analyze than other physiological signals. There are many studies performed by obtaining pulse information and ECG signals from patients in intensive care [8-11]. Most of these studies are for the aim of establishing or developing alarm systems [12-14]. The ECG reflects the electrical activity of the heart controlled by the autonomic nervous system. When a person faces a situation that causes stress, the sympathetic nervous system occurs dominant in the autonomic nervous system and increases the heart rate. With the parasympathetic system, the body is attained to balance by reducing the heart rate [15].

The aim of this study is to evaluate the contribution of ECG signals in the follow-up of for improving physiological well-being patients in coma in Intensive Care Units (ICU). For this reason, ECG signals were obtained from three coma patients on different days, during the interaction between the nurse and the family, and at rest. Attribute extraction is done from ECG signals. Thus, it was tried to obtain information about the patient's well-being. As a result, it was shown that patients react to their relatives and nurses even if they are in a coma and monitoring of their health status can be done with ECG signals.

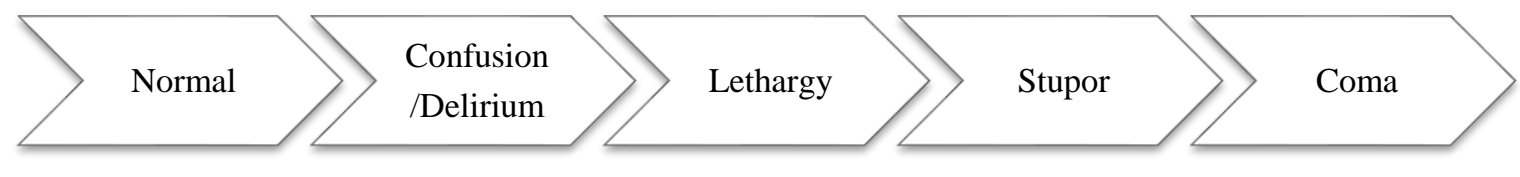

Figure 1. Level of consciousness [3]. 


\section{Materials and Method}

\subsection{Recording System}

After obtaining the approval of the Ethics Committee for our study, ECG signals were examined in different scenarios on the days determined by the physician from three patients with Glasgow Coma Scale between 3-10. As shown in Figure 2, ECG recordings were obtained from single channel (derivation I) using Biopac MP-150 device with a sampling frequency at $1000 \mathrm{~Hz}$. Records were taken at five events: rest, interaction with the family and nurse. ECG signals were recorded at approximately 35 minutes duration and the performed process was defined in the below flow chart. In the event of rest, no stimulus was given to the patient and the environment was kept as quiet as possible. During the interaction with the nurse, the patient wanted to make certain commands such as opening his eyes and moving his arm. During the interaction with the family, personal information of the family were shared with the patient and provided contact with patient's hands. Also nurse and relatives of patients spoke to patients. Table 1 shows the demographic information for patients analyzed in this study.

* 5 minutes of rest recording (first basal event)

- 5 minutes record during nurse interaction event

* Rest for 10 minutes (second basal event)

* 5 minutes recording during family visit (family interaction event)

* Repeat baseline for 10 minutes (final/third basal event)

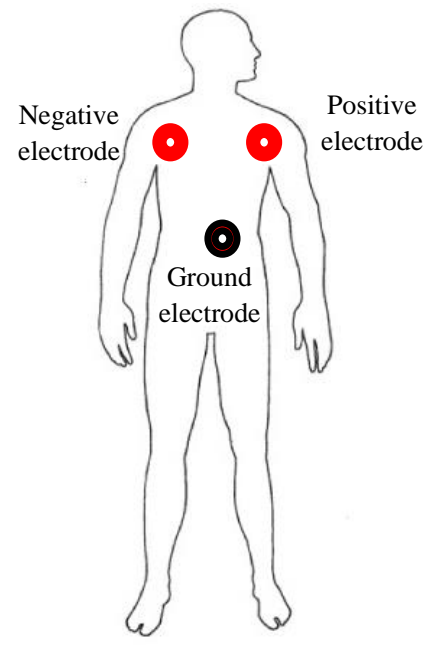

Figure 2. Electrode placement for ECG

Table 1. Demographic informations of patients

\begin{tabular}{llccc}
\hline & Gender & Age & Stay Time (Day) & GCS \\
\hline First Patient & Female & 85 & -19 & 5 \\
\hline Second Patient & & & 40 & 5 \\
& Male & 34 & -36 & 8 \\
\hline Third Patient & & & 47 & 5 \\
& Female & 56 & -34 & 6 \\
\hline
\end{tabular}




\subsection{Attributes}

The ECG signals were filtered using $4^{\text {th }}$ Degree low pass butterworth filter with $100 \mathrm{~Hz}$ cut off frequency and $50 \mathrm{~Hz}$ notch filter (to eliminate mains voltage). Then, heart rate variability (HRV) was analyzed in time domain. HRV is calculated by evaluating at the time differences between each $\mathrm{R}$ wave in the ECG signal. In order to obtain HRV, it is necessary to find the time interval between the $\mathrm{R}$ waves of the QRS components in the ECG signals. The attributes obtained from ECG signals in this study are shown in Table 2. MATLAB program was used to extract the attributes.

Table 2. Attributes

\begin{tabular}{cc}
\hline Attribute & Definition \\
\hline RR_STD & Standard deviation of R-R intervals \\
RR_RMS & Root mean squares of R-R intervals \\
AVG_BPM & Mean heart rate \\
ECG_MEAN & Mean value of ECG signal \\
ECG_STD & Standard deviation of ECG signal \\
ECG_RMS & Root mean squares of ECG signal \\
\hline
\end{tabular}

The standard deviation is a measure that uses the distribution of numbers in a series around the average of that series to summarize the spread of the data values and is calculated as follows:

$$
s t d=\sqrt{\frac{\sum_{i=1}^{N}\left(X_{i}-\bar{X}\right)^{2}}{N-1}}
$$

Root Mean Square (RMS) returns the effective value of the signal and is the square root of the average of the sum of the squares of its elements (Equation 2). It is a statistical criterion used to measure the magnitude of varying amounts.

$$
R M S=\sqrt{\frac{1}{N} \sum_{i=1}^{N} X_{i}^{2}}
$$

\subsection{Statistical Test}

The Wilcoxon signed rank test, which is a non-parametric test, was used for statistical analysis of the data obtained in order to observe the changes in the health condition of the patients. This method is used to observe the differences between the two related groups. For example, it is applied to analyze the changes in the average pulse in the ECG signal in case of first rest and family interaction [16]. In this study, it was used to examine changes in first rest-family, familysecond rest, second rest-nurse, nurse-last rest events using obtained attributes. It was also used to investigate at the change in health status of patients in the ICU. In this study, Wilcoxon signed rank test was preferred because of the low number of data and not having normal distribution. 


\section{Results}

The results were compared between events to measure the patient's response to the family and the nurse. The events examined are indicated below. Figure 3 shows the events that are compared. ECG recordings were taken on two different days from 3 patients. Thus, a total of 6 ECG signals were analyzed. Attributes were calculated separately for 5 events: first basal, family, second basal, nurse, third basal. Thus, 30 instances were obtained in the study. 28 samples were examined because the nurse and final resting conditions could not be analyzed for one patient.

First basal-family interaction

$>$ Family interaction-second basal

$>$ Second basal-nurse interaction

$>$ Nurse interaction-third basal

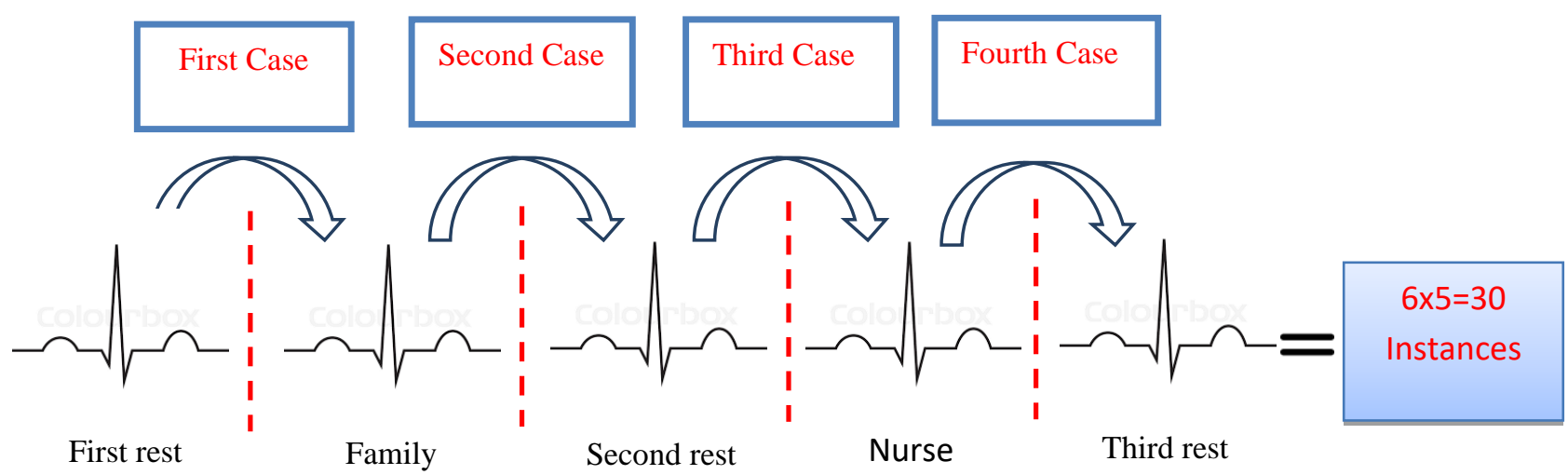

Figure 3. Comparison groups in statistical analysis

\subsection{First basal- Family interaction events}

Table 3 shows the descriptive statistics of the two events. This table contains information such as mean, standard deviation, maximum and minimum values of the obtained attributes. Table 4 shows the changes of attributes between events. Negative rank indicates the number of people who have experienced a decrease in related attribute value. The positive rank indicates the number of people who have experienced an increase in related attribute value. The same rank indicates the number of people who have unchanged value in related attribute. For example, according to the AVG_BPM attribute, there are 1 people whose average pulse decreases during communication with the family and 5 people whose average pulse increases during communication with the family. Mean heart rate increases during interaction with the family. When the average of AVG_BPM attribute is examined in Table 3, the mean pulse rate is 98 in the first basal event and 105 in the family interaction. It was observed that during the person's family talk and touching it is caused the heart to beat faster by activating the sympathetic system. 
Table 3. Descriptive statistics for first basal-family interaction events

\begin{tabular}{ccccc}
\hline Attribute & Mean & $\begin{array}{c}\text { Standard } \\
\text { Deviation }\end{array}$ & Minimum & Maximum \\
\hline \multicolumn{5}{c}{ First Basal Values } \\
\hline RR_STD & 3.849 & 8.659 & 0.01 & 21.50 \\
RR_RMS & 5.569 & 12.340 & 0.00 & 30.71 \\
AVG_BPM & 98.700 & 9.407 & 82.78 & 108.40 \\
ECG_MEAN & 0.022 & $0 . .309$ & 0.00 & 0.08 \\
ECG_STD & 0.147 & 0.064 & 0.06 & 0.22 \\
ECG_RMS & 0.015 & 0.007 & 0.01 & 0.02 \\
\hline \multicolumn{5}{c}{ Family Interaction Values } \\
\hline RR_STD & 3.849 & 8.659 & 0.01 & 21.50 \\
RR_RMS & 15.280 & 27.895 & 0.02 & 71.02 \\
AVG_BPM & 105.415 & 15.575 & 82.87 & 130.42 \\
ECG_MEAN & 0.027 & 0.041 & 0.00 & 0.10 \\
ECG_STD & 0.148 & 0.062 & 0.07 & 0.22 \\
ECG_RMS & 0.015 & 0.007 & 0.01 & 0.02 \\
\hline
\end{tabular}

Table 4. Ranks of First basal-family interaction events

\begin{tabular}{|c|c|c|c|c|c|c|}
\hline & RR_STD & RR_RMS & AVG_BPM & ECG_MEAN & ECG_STD & ECG_RMS \\
\hline Negative Rank $^{a}$ & 0 & 2 & 1 & 4 & 3 & 3 \\
\hline Positive Rank ${ }^{\mathbf{b}}$ & 0 & 4 & 5 & 2 & 3 & 3 \\
\hline Same Rank ${ }^{c}$ & 6 & 0 & 0 & 0 & 0 & 0 \\
\hline
\end{tabular}

\subsection{Family interaction-second basal events}

Table 5. Descriptive statistics for family interaction-second basal events

\begin{tabular}{ccccc}
\hline Attribute & Mean & $\begin{array}{c}\text { Standard } \\
\text { Deviation }\end{array}$ & Minimum & Maximum \\
\hline \multicolumn{5}{c}{ Family Interaction Values } \\
\hline RR_STD & 3.849 & 8.659 & 0.01 & 21.50 \\
RR_RMS & 15.280 & 27.895 & 0.02 & 71.02 \\
AVG_BPM & 105.415 & 15.575 & 82.87 & 130.42 \\
ECG_MEAN & 0.027 & 0.041 & 0.00 & 0.10 \\
ECG_STD & 0.148 & 0.062 & 0.07 & 0.22 \\
ECG_RMS & 0.015 & 0.007 & 0.01 & 0.02 \\
\hline \multicolumn{5}{c}{ Second Basal Values } \\
\hline RR_STD & 3.849 & 8.659 & 0.01 & 21.50 \\
RR_RMS & 2.012 & 3.638 & 0.01 & 9.06 \\
AVG_BPM & 105.761 & 17.464 & 84.37 & 134.45 \\
ECG_MEAN & 0.028 & 0.041 & 0.00 & 0.10 \\
ECG_STD & 0.149 & 0.062 & 0.07 & 0.22 \\
ECG_RMS & 0.015 & 0.007 & 0.01 & 0.02 \\
\hline
\end{tabular}

Table 6. Ranks of family interaction-second basal events

\begin{tabular}{c|c|c|c|c|c|c}
\hline & RR_STD & RR_RMS & AVG_BPM & ECG_MEAN & ECG_STD & ECG_RMS \\
\hline $\begin{array}{c}\text { Negative } \\
\text { Rank }^{\mathrm{a}}\end{array}$ & 0 & 5 & 2 & 2 & 2 & 2 \\
\hline
\end{tabular}




\begin{tabular}{c|c|c|c|c|c}
$\begin{array}{c}\text { Positive } \\
\text { Rank }^{\mathbf{b}}\end{array}$ & 0 & 1 & 4 & 4 & 4 \\
\hline $\begin{array}{c}\text { Same } \\
\text { Rank }^{\mathbf{c}}\end{array}$ & 6 & 0 & 0 & 0 & 0 \\
\hline a. Family>second basal, b. Second basal>family, c. Family=second basal & & 0 \\
\hline
\end{tabular}

\subsection{Second basal-nurse interaction events}

Table 7. Descriptive statistics for second basal-nurse interaction events

\begin{tabular}{ccccc}
\hline Attribute & Mean & $\begin{array}{c}\text { Standard } \\
\text { Deviation }\end{array}$ & Minimum & Maximum \\
\hline \multicolumn{5}{c}{ Second Basal Values } \\
\hline RR_STD & 4.535 & 9.497 & 0.01 & 21.50 \\
RR_RMS & 2.411 & 3.917 & 0.01 & 9.06 \\
AVG_BPM & 100.023 & 11.591 & 84.37 & 109.95 \\
ECG_MEAN & 0.032 & 0.044 & 0.00 & 0.10 \\
ECG_STD & 0.151 & 0.069 & 0.07 & 0.22 \\
ECG_RMS & 0.016 & 0.008 & 0.01 & 0.02 \\
\hline \multicolumn{5}{c}{ Nurse Interaction Values } \\
\hline RR_STD & 4.535 & 9.497 & 0.01 & 21.50 \\
RR_RMS & 1.743 & 2.874 & 0.00 & 6.65 \\
AVG_BPM & 105.929 & 22.882 & 80.60 & 141.70 \\
ECG_MEAN & 0.027 & 0.032 & 0.00 & 0.07 \\
ECG_STD & 0.152 & 0.063 & 0.08 & 0.22 \\
ECG_RMS & 0.015 & 0.007 & 0.01 & 0.02 \\
\hline
\end{tabular}

Table 8. Ranks of second basal-nurse interaction events

\begin{tabular}{|c|c|c|c|c|c|c|}
\hline & RR_STD & RR_RMS & AVG_BPM & ECG_MEAN & ECG_STD & ECG_RMS \\
\hline $\begin{array}{c}\text { Negative } \\
\text { Rank }^{\mathbf{a}}\end{array}$ & 0 & 4 & 3 & 2 & 3 & 3 \\
\hline $\begin{array}{c}\text { Positive } \\
\text { Rank }^{\mathbf{b}}\end{array}$ & 0 & 1 & 2 & 3 & 2 & 2 \\
\hline $\begin{array}{l}\text { Same } \\
\text { Rank }^{c}\end{array}$ & 5 & 0 & 0 & 0 & 0 & 0 \\
\hline
\end{tabular}

\subsection{Nurse interaction-third basal}

Table 9. Descriptive statistics for Nurse interaction-third basal events

\begin{tabular}{ccccc}
\hline Attribute & Mean & $\begin{array}{c}\text { Standard } \\
\text { Deviation }\end{array}$ & Minimum & Maximum \\
\hline \multicolumn{5}{c}{ Nurse Interaction Values } \\
\hline RR_STD & 4.535 & 9.497 & 0.01 & 21.50 \\
RR_RMS & 1.743 & 2.874 & 0.00 & 6.65 \\
AVG_BPM & 105.929 & 22.882 & 80.60 & 141.70 \\
ECG_MEAN & 0.027 & 0.032 & 0.00 & 0.07 \\
ECG_STD & 0.152 & 0.063 & 0.08 & 0.22 \\
ECG_RMS & 0.015 & 0.007 & 0.01 & 0.02 \\
\hline \multicolumn{5}{c}{ Third Basal Values } \\
\hline RR_STD & 4.535 & 9.497 & 0.01 & 21.50 \\
RR_RMS & 2.472 & 4.241 & 0.01 & 9.83
\end{tabular}




\begin{tabular}{ccccc} 
AVG_BPM & 97.735 & 11.158 & 79.66 & 107.69 \\
ECG_MEAN & 0.028 & 0.035 & 0.00 & 0.08 \\
ECG_STD & 0.146 & 0.064 & 0.07 & 0.21 \\
ECG_RMS & 0.015 & 0.007 & 0.01 & 0.02 \\
\hline
\end{tabular}

Table 10. Ranks of Nurse interaction-third basal events

\begin{tabular}{c|c|c|c|c|c|c}
\hline & RR_STD & RR_RMS & AVG_BPM & ECG_MEAN & ECG_STD & ECG_RMS \\
\hline $\begin{array}{c}\text { Negative } \\
\text { Rank }\end{array}$ & 0 & 0 & 4 & 2 & 4 & 4 \\
\hline $\begin{array}{c}\text { Positive } \\
\text { Rank }^{\mathbf{b}}\end{array}$ & 0 & 5 & 1 & 3 & 1 & 1 \\
\hline $\begin{array}{c}\text { Same } \\
\text { Rank }^{\mathbf{c}}\end{array}$ & 5 & 0 & 0 & 0 & 0 & 0 \\
\hline
\end{tabular}

\section{Discussion}

When Table 5 and Table 6 were examined, it was observed that patients' heart rate increased after family visit. In the RR_RMS attribute, there was a significant decrease in rest after the family (second rest). The RMS value of the ECG signal decreased at second rest. When Table 7 and Table 8 are examined, it is seen that nurse interaction has a calming effect due to decreases in standard deviations. Thus, it can be thought that nurse interaction increases parasympathetic activity. Because they are experienced, they have an important role in healing the patient.

In addition, the first patient was evaluated as GCS 5 on the 19th day and on the 40th day. On day 19 , the pulse rate was around $80 \mathrm{bpm}$ (beat per minute), while on day 40 it was about $100 \mathrm{bpm}$. In addition, while the ECG_STD attribute was 0.09, it was around 0.06 on day 40. The standard deviation of the ECG wave was reduced. She had a higher heart rate on day 19 and a more regular ECG wave on day 40. However, the standard deviation of R waves (RR_STD) increased approximately 20 -fold compared to day 19 . With these data, it is seen that the patient has more irregular heartbeat, ie the patient's health condition does not improve.

When second patient was evaluated, the GCS was 8 on the day 36 and the GCS was 10 on the day 47. The average pulse was $130 \mathrm{bpm}$ on day 36, while it was around $108 \mathrm{bpm}$ on day 47. The standard deviation of R waves (RR_STD) 50 times lower on day 47 compared to day 36 . In other words, it can be evaluated that the patient's health condition improves because he has more regular heartbeats and a lower pulse rate.

The third patient was evaluated as GCS 5 on day 6 and GCS 6 on day 34 in the ICU. The average pulse was $105 \mathrm{bpm}$ on day 6 and $95 \mathrm{bpm}$ on day 34. Although the standard deviation of ECG waves and the standard deviation of R-R waves are similar for day 6 and day 34, RR_STD decreased by 1.26 times on day 34. For this patient, whose consciousness level increased, her health condition was in the direction of recovery. 


\section{Conclusions}

In this study, 3 patients with GCS 3-10 hospitalized in the ICU were examined with ECG signals recorded in different scenarios on different days. The main conclusions of the study, the changes in the health condition of the patients can be defined according to the attributes obtained from ECG signals recorded in the proposed scenarios of comatose patients. In addition, it has been shown that patient follow-up can be easily performed using ECG signals. Attributes of ECG signals and consciousness levels were correlated to obtain information about the patient's condition to help the physician. This is a case study carried out with 3 patients. Henceforward, it is planned to increase the number of data and obtain more precise results in patient follow-up.

\section{Acknowledgements}

This study was supported by Erciyes University Scientific Research Projects Unit (Project number: FDK-2017-7531).

\section{References}

[1] Cooksley V, T Holland, The unconscious patient, Medicine. 2013;41(3): 146-150.

[2] Campbell S, McCormick W. Approach to the comatose patient. Can JCME 2002; 77-84.

[3] Sepit D. Bilinç durumunun değerlendirilmesi ve Glasgow Koma skalası. Hemşirelikte Eğitim ve Araştırma Dergisi 2005; 2(1): 12-6.

[4] Flower L. Literature Survey on Biomedical Signal Processing Methods. International Journal of Innovative Research in Computer and Communication Engineering 2016; 4(2): 50-4.

[5] Wieser M, Koenig B.A, Riener R. Quantitative Description of the State of Awareness of Patients in Vegetative and Minimally Conscious State. 32nd Annual International Conference of the IEEE EMBS Buenos Aires, Argentina,5533-5536, August 31 - September 4, 2010.

[6] Rigas G, Goletsis Y, Fotiadis DI. Real-Time Driver's Stress Event Detection. IEEE Transactions on Intelligent Transportation Systems 2012; 13:221-234.

[7] Begum S., Ahmed MU, Funk P, Xiong N, Schéele B.V. Case-Based Decision Support System for Individual Stress Diagnosis Using Fuzzy Similarity Matching. The Journal of Computational Intelligence (CI), 2009; 25(3):180-195.

[8] Arslan S, Ozer N. Touching, Music Therapy and Aromatherapy's Effect on the Physiological Situation of the Patients in Intensive Care Unit. International Journal of Caring Sciences 2016; 9 (3):867.

[9] Chan MF. et al. Effects of music on patients undergoing a Cclamp procedure after percutaneous coronary interventions. Journal of Advanced Nursing 2006; 53(6): 669-679.

[10] Cooke B, Ernst E. Aromatherapy: a systematic review. British Journal of General Practice 2000; 50: 493-6.

[11] Bashar SK, Ding E, Walkey AJ, McManus DD, Chon KH. Noise Detection in Electrocardiogram Signals for Intensive Care Unit Patients. IEEE Access, 2019;7:8835788368. 
[12] Lin C, Chen S, Wang Y, Lu S. Develop a multiple physiological system of ICU patients with symptom analysis and decision making. 2014 IEEE International Conference on Consumer Electronics - Taiwan, Taipei, 2014; 163-164.

[13] Serackis A, Abromavičius V, Gudiškis A. Identification of ECG signal pattern changes to reduce the incidence of Ventricular Tachycardia false alarms. 2015 Computing in Cardiology Conference (CinC), Nice, 2015;1193-1196.

[14] Manna T, Swetapadma A. An Improved Method for Detecting False Alarm in Arrhythmia ICU Patients. 2018 Fourth International Conference on Research in Computational Intelligence and Communication Networks (ICRCICN), Kolkata, India, 2018; 66-69.

[15] Oh B.S, Yeo Y. K, Wan F. Y, Wen Y, Yang Y, Lin Z. Effects of noisy sounds on human stress using ECG signals: An empirical study. In 2015 10th International Conference on Information, Communications and Signal Processing (ICICS) 2015; 1-4.

[16] Kocaçalışkan İ, Akanıl Bingöl N. Biyoistatistik, $2^{\text {nd }}$ ed. pp. 184, 2008. 